\title{
Automatic Orthographic Road Image Generation Using in Vehicle Sensors for ADAS
}

\author{
Junichi Meguro $^{1)}$ Hiroyuki Ishida $^{2)}$ Chunzhao Guo $^{3)}$ Koichiro Yamaguchi4) $^{(4)}$ Yoshiko Kojima $^{5)}$ \\ 1)-5) Toyota Central R\&D Labs., Inc., \\ 41-1 Yokomichi, Nagakute City, Aichi Pref., Japan \\ E-mail:meguro@mosk.tytlabs.co.jp
}

Received on March, 17,2015

Presented at the AVEC'14 on September 25, 2014

\begin{abstract}
This paper proposes a technique to generate orthographic road images and lane-level road maps suitable for advanced driver assistance systems (ADAS) using in-vehicle low-cost sensors, i.e., a single-frequency GPS, speed sensor, yaw rate gyroscope, and rear-view camera. This research also aims to generate accurate digital maps from vehicle driving data using the probe technique. This proposal should help to reduce the production costs of accurate digital maps that include road markings and road networks. The key feature of this method is the integration of probe data based on accurate trajectories and heading angles to generate digital maps. Comparisons between commercial orthographic road images and our proposals show that the proposed method automatically generates an orthographic image with a similar precision to that of the survey level method of commercial ones.
\end{abstract}

KEY WORDS: Electronics and control, Autonomous driving system, Image processing / Digital Map, Positioning [E1]

\section{Introduction}

Recently, various advanced driver assistance systems (ADAS) have been developed. Driver assistance systems that alert the driver before a stop sign, intersection, and the like and vehicle to infrastructure cooperative systems are attracting attention. In addition, some autonomous car projects are going on. The efficiency of such systems fundamentally depends on accurate vehicle localization and high-cost digital maps ${ }^{(1-3)}$. Ziegler proposed localization technique for autonomous car using camera and self-developed map ${ }^{(3)}$. The map is created by using the invehicle camera images and accurate IMU. The self-developed map are used to not only localization but also control and navigation. The distance between autonomous vehicle and road boundary or white line are important information to control vehicle. Localization/potining and map generation are necessary technique to realize ADASs using map that include autonoumous car.

Therefore, we developed a new positioning technique ${ }^{(4)}$ that uses accurate vehicle trajectory ${ }^{(5)}$. These techniques can realize accurate positioning using low-cost sensors (a standard GPS, lowcost yaw-rate gyro, and speed sensor). However, accurate and low cost digital maps are not currently available. Conventionally, accurate maps are commercially produced and require large costs. Consequently, this paper proposes a technique to generate orthographic road images and lane level digital map, that are suitable for ADAS, using low cost sensor data. This technique needs large ammount of vehicle data include image. This issue will be sloved by probe technique in near future.

\section{Related Works}

Several accurate digital map generation techniques have been proposed for road object survey and management ${ }^{(6-7)}$. Some of these have already been commercialized. However, cost remains a serious challenge in applying these techniques to ADAS. There are two reasons for this high cost. The first is the cost of the measurement systems. Most use mobile mapping systems (MMS) that require expensive sensors. These MMSs use accurate GPS/INS (a combination of a two-frequency GPS, accurate gyro, and speed sensor), cameras, and lidars. MMSs have been used to carry out many accurate and precise surveys of roads, bridges, and buildings. However, the GPS/INS that enable positioning with error of less than a few meters in city areas, are expensive since highly accurate gyros are required. This makes low-cost digital map generation difficult. The second reason is that conventional digital map creation requires a large number of work hours. In most cases, accurate digital maps cannot tolerate any mistakes and require manual checking to enhance reliability.

At the same time, much research has been proposed related to ITS using probe technology, which collects large amounts of data ${ }^{(8-10)}$. Shladover estimated the state of the road surface from data collected from vehicles, and $\mathrm{Li}$ calculated the travel time ${ }^{(8)}$. Some commercial car navigation systems provide updated information on newly constructed roads quickly using probe technology. There is also service that uploads camera images to share traffic information ${ }^{(9)}$.

Therefore, this paper proposes a method to reduce the generation cost of accurate digital maps using vehicle driving data. The method focus on generating road-images with low cost sensors using the probe technique. Use of such road images should help to lower the cost of digital maps. In addition, it should also enhance development of more advanced ADAS applications. Several techniques have been proposed for generating roadimages through image mosaicing, using one or more on-board cameras $^{(11-12)}$. However, a technique for generating a wide range of orthographic images has not yet been proposed. Geiger 


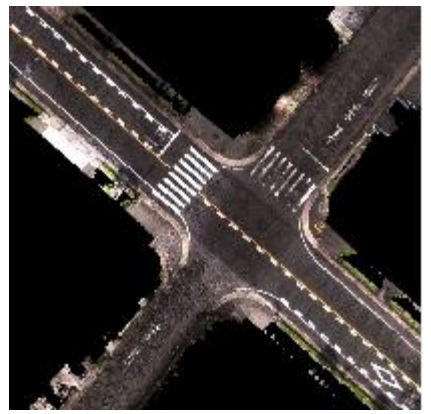

(a) Orthographic road image

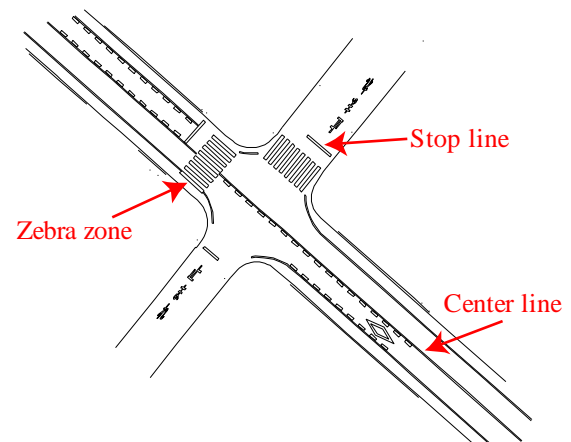

(b) Survey level ditital road map

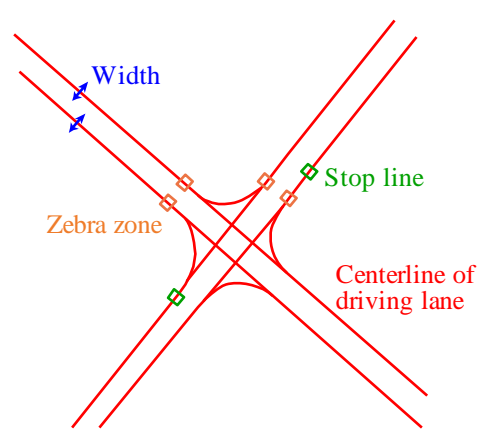

(c) Lane level map

Fig. 1 Examples of orthographic road image, survey level digital road map and lane level map.

proposed an image generation technique that mosaics images captured by other vehicles for filling in blind areas ${ }^{(11)}$. However, this technique mosaics only a narrow range of images. Widerange image mosaicing is difficult because this technique does not utilize position information. Noda proposed a road-image generation technique based on accurate orthographic aerial images $^{(12)}$. However, this techniques for generating maps needs aerial images. In addition, image feature matching are affected by other vehicles.

\section{Overview}

This paper proposes a method to generate orthographic road images using low-cost in-vehicle sensors. Lane level maps can then be constructed automatically based on the integrated road images and real vehicle trajectories. Fig. 1 shows examples of road image and lane level map. An example of survey level road digital map is also shown in Fig. 1. Lane-level maps consist of the centerline of the driving lane associated with additional information such as width, curvature, and the like. The connections of each driving lane are also included in a lane-level map.

The key feature of our proposal is the integration of multiple items of vehicle driving data based on accurate trajectories and heading angles. Fig. 2 gives an overview of the proposal. One vehicle collects sensor data for generating a local area map using information, for one lane, and short distances $(100 \mathrm{~m})$. An accurate map for a large area can be obtained by integrating local maps collected by several vehicles.

This technique uses a low-cost (single-frequency) GPS receiver, an inertial navigation system (INS) that includes a speed sensor and low-cost yaw-rate gyroscope, and a rear-view camera. The vehicle trajectory and heading angle are estimated mainly
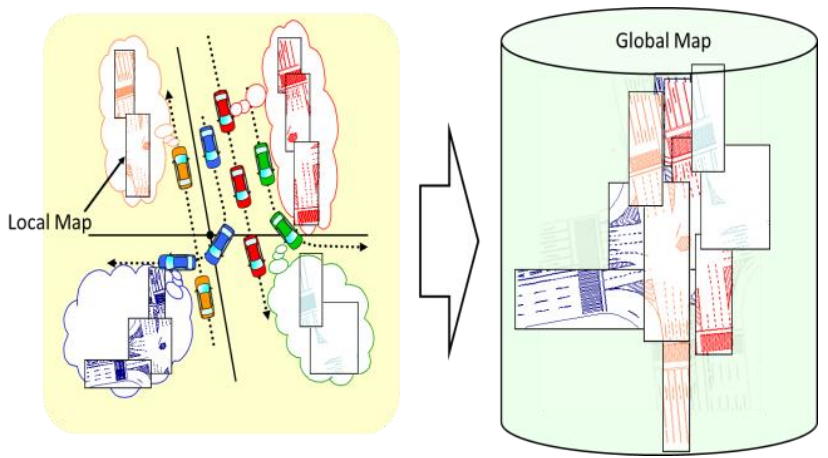

Fig. 2 Overview of our proposal.

from the Doppler shift of the GPS signal (i.e., the GPS Doppler). In places, GPS signals are not blocked from overhead, an accurate vehicle trajectory and heading angle can be estimated using the GPS Doppler. This feature enables simple map generation with a combination of devices that are less expensive than current map generation techniques.

Fig. 3 shows the proposed road map generation flow. Road images are created by projecting rear-view camera images onto the road surface along the vehicle trajectory. An orthographic road image is an image that shows the road surface from above. Consequently, an orthographic image is generated by integration with the road image. Lane-level maps for ADAS can be generated from orthographic road images with image processing.

\section{GPS Doppler Based Trajectory Estmation}

Vehicle position and trajectory accuracy are important for automatic map generation. In addition, this proposal also assumes the use of low-cost sensors. The GPS Doppler is used for the

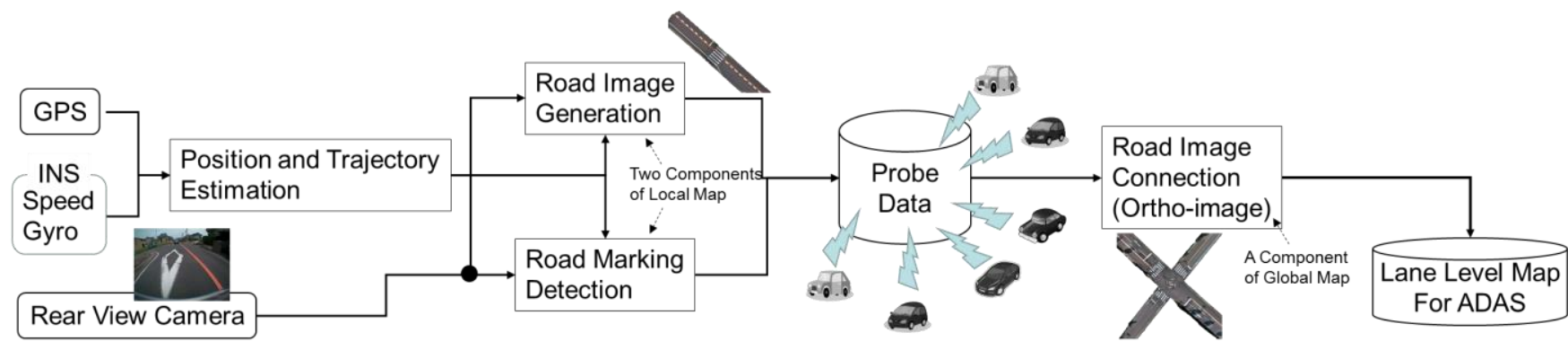

Fig. 3 Proposed ADAS road map generation flow. 
vehicle trajectory and heading angle estimation. Fig. 4 shows an overview of the GPS Doppler. GPS Doppler processing obtains the relative velocity between a GPS satellite and a receiver, and calculates the velocity from the frequency shift of the carrier signal. Then, the velocity vector of the vehicle is calculated based on the GPS Doppler, and the heading angle is obtained from the velocity vector. One feature of this method is that sensor error does not accumulate with successive heading estimations $^{(12)}$. This feature is suitable for the generation of road images. Fig. 5 shows velocity estimation results using GPS Doppler that is measured in an open sky environment. These results indicate that the GPS Doppler can estimate velocity accurately. The error is $0.2 \mathrm{~m} / \mathrm{s}$ (standard deviation (SD)).

However, multi-path reflections and blocking of GPS signals occur in urban areas, which reduce the accuracy of velocity data. Also, the GPS Doppler requires four satellites to estimate vehicle velocity. For this reason, Takeyama proposed a heading estimation technique that improves trajectory accuracy by tight coupling of time-series GPS-Doppler and $\operatorname{INS}^{(5)}$. This proposal improves the accuracy of trajectory estimation in urban areas. The velocity accuracy is $1.0 \mathrm{~m}$ per $100 \mathrm{~m}$ in rural areas and $2.5 \mathrm{~m}$ per $100 \mathrm{~m}$ in urban areas.

In our proposal, we collect vehicle driving data with trajectory that is estimated by the technique ${ }^{(5)}$. Fig. 6 (a) shows the position and trajectory estimation results of the three vehicles, which were obtained at different times and dates. In addition, sensors from different manufacturers were used. The

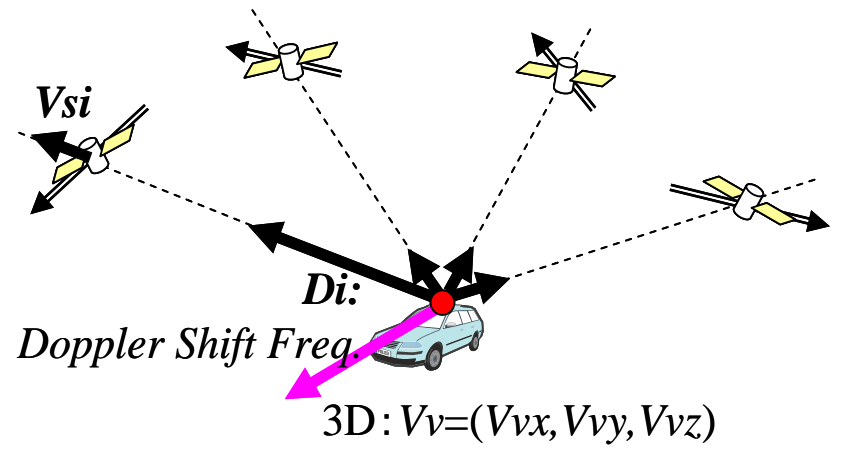

Fig. 4 Overview of GPS Doppler.

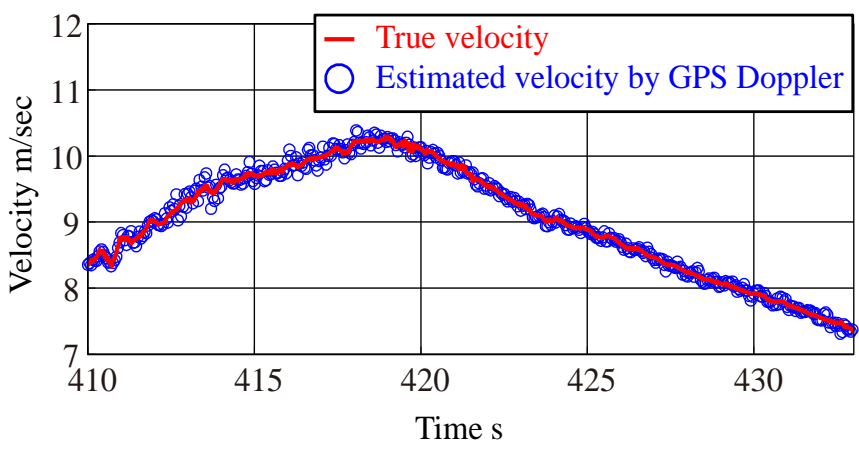

Fig. 5 Velocity by GPS Doppler.
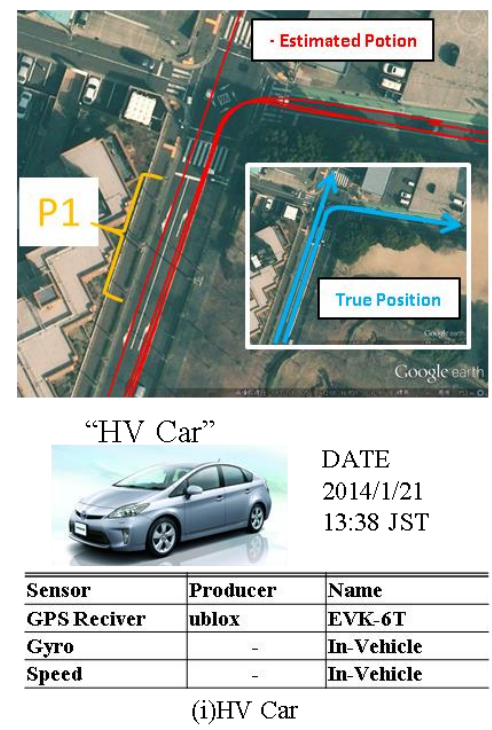
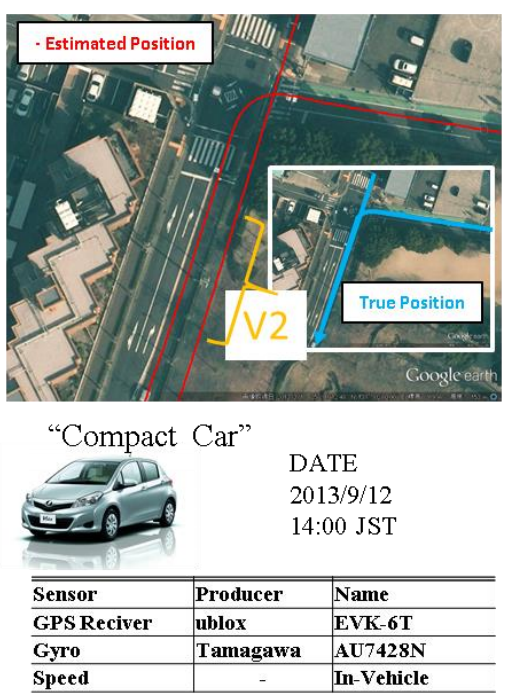

(ii)Compact Car
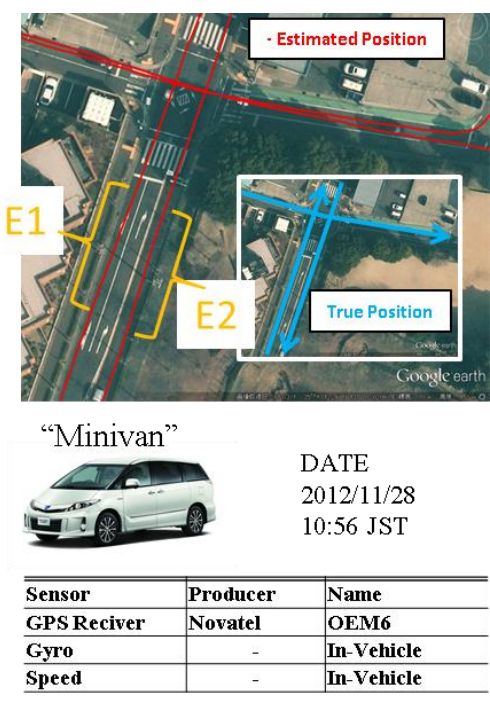

(iii)Minivan

(a) Data description of HV Car, Compact Car and Minivan.

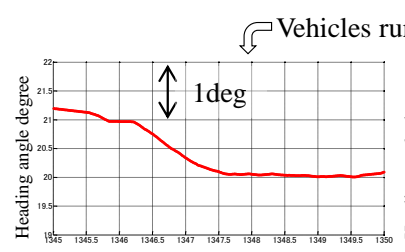

Time sec

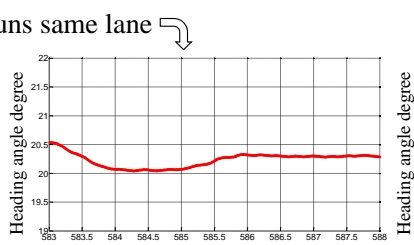

Time sec

(b) Comparisons of heading angles in same lane.

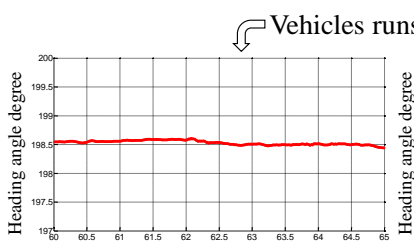

Time sec
(iii)Heading angle at V2

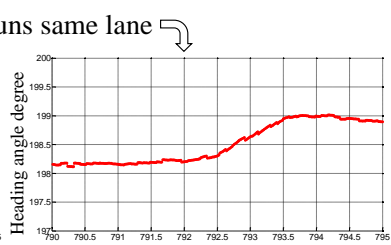

Time sec
(iv)Heading angle at E2

Fig. 6 Comparison of vehicle position, trajectory and heading angle. 
same position and direction were estimated for the HV Car (P1), Minivan (E1), Compact Car (V2), and Minivan (E2). Fig. 6 (b) compares the heading angles at P1, E1, V2, and E2. These results show that this proposal enables to estimate almost same heading angles and trajectory shapes for different vehicles driving in the same lane. Fig.6 (b) shows each vehicle's heading angle accuracy seems to be less than 1 degree. This accurate heading angle and trajectory helps to integrate the collected vehicle driving data using the probe technique. The trajectory error is less than $1 \mathrm{~m}$ per $100 \mathrm{~m}$ distance. Heading accuracy less than 1degree is equivalent to the target trajectory accuracy in our proposal. Accurate heading angle reduces rotation search rage at the process of road image generation.

\section{Road Image Generation}

A road image illustrates road serface that is look like orthografic images. Meguro proposed road image generation techniue by connecting rear view camera images in accordance with the vehicle trajectory ${ }^{(13-14)}$. Fig. 7 shows an overview of road image generation. A road image of the road surface can be obtained by overwrapping bird's-eye view images shifted by parallel translation onto the center of each road image, which is moved according to the vehicle's trajectory. Accurate trajectory estimation enables precise image projection without the tracking of features in the camera images.

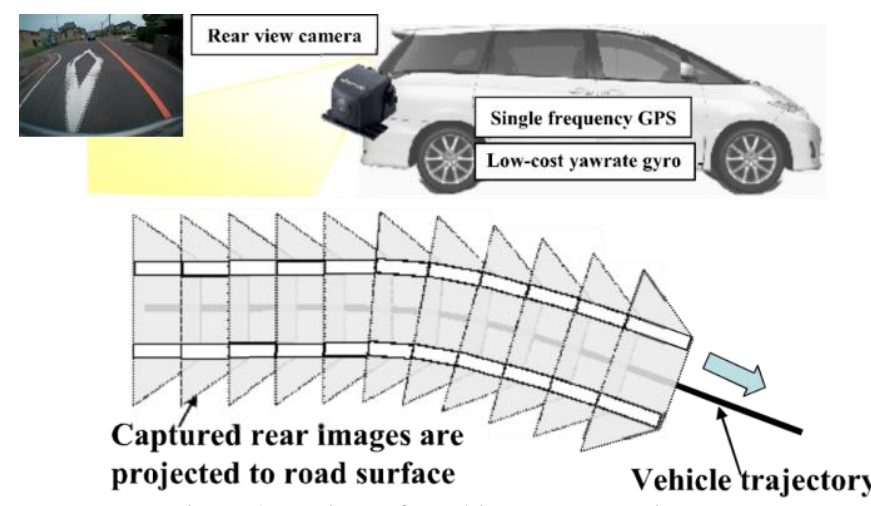

Fig. 7 Overview of road image generation.

Fig. 8 compares a road image generated with this proposal and a road image generated by a conventional positioning and heading estimation technique. This conventional technique uses a loosely coupled (LC) GPS/INS technique that integrates the GPS positioning results, the gyro yaw rate angle, and vehicle speed using a Kalman Filter ${ }^{(15)}$. In Fig. 8, the road image generated by the LC GPS/INS positioning and heading estimation results was distorted by positional and heading errors. The major factor influencing these errors is the GPS multi-path ${ }^{(4)}$. In contrast, the proposed trajectory and heading estimation technique were unaffected by the GPS multi-path. This advantage enables the generation of precise road images.

Fig. 9 shows generated road images that form a lane. Road objects (the road signs, zebra crossing, and the like) are shown precisely. In addition, these road images are tagged with accurate heading angles. Road images that show several lanes are made by connecting road images. In addition, Fig. 10 shows position error of road images at straight road. Each color line at Fig.10 (a) and Fig.10 (b) shows same run's trajectory error. 5 data are shown in Fig.10. Along and across position errors of road images are less than $0.3 \mathrm{~m}$ in 100 meters distance.

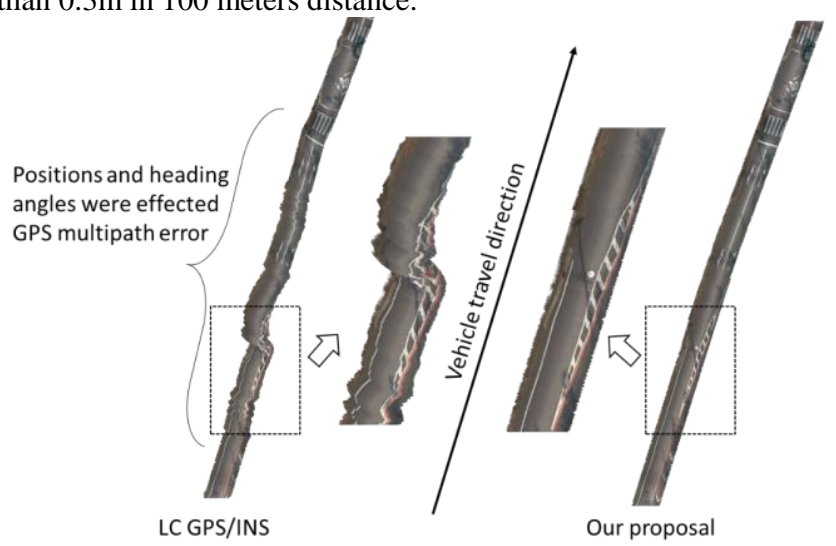

Fig. 8 Comparison between the proposed technique and conventional positioning and heading estimation technique.

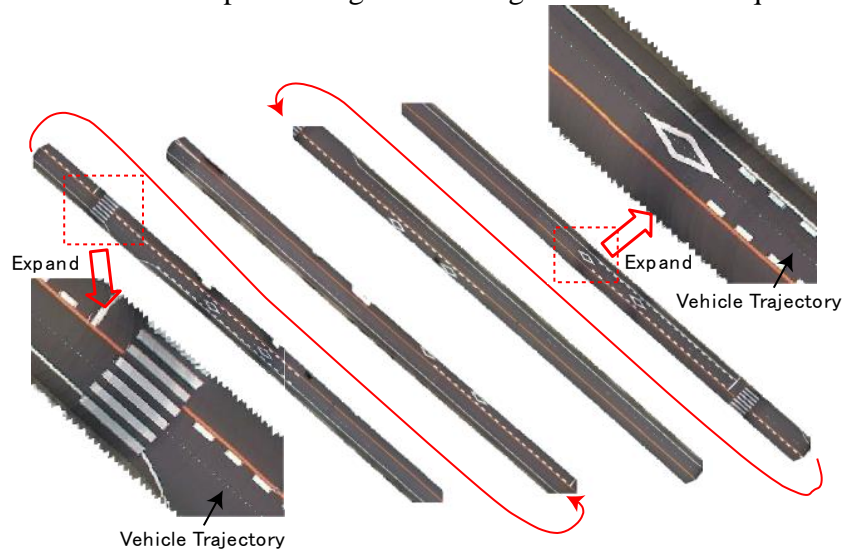

Fig. 9 Road images by vehicle trajectory.

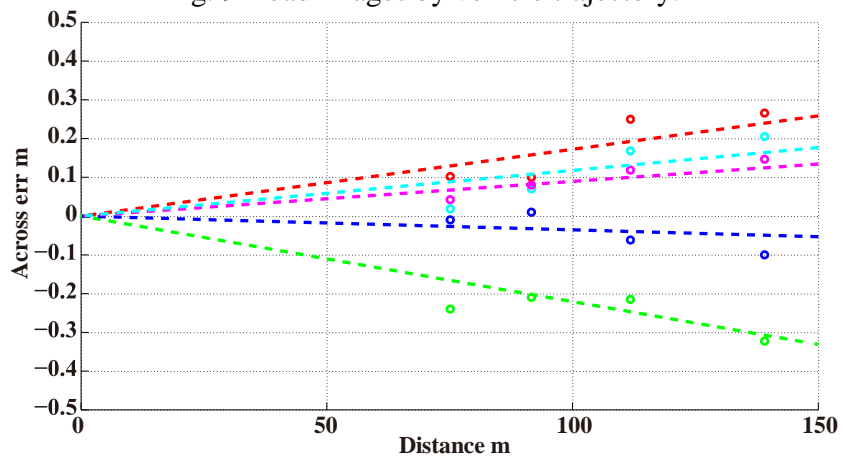

(a)Across error

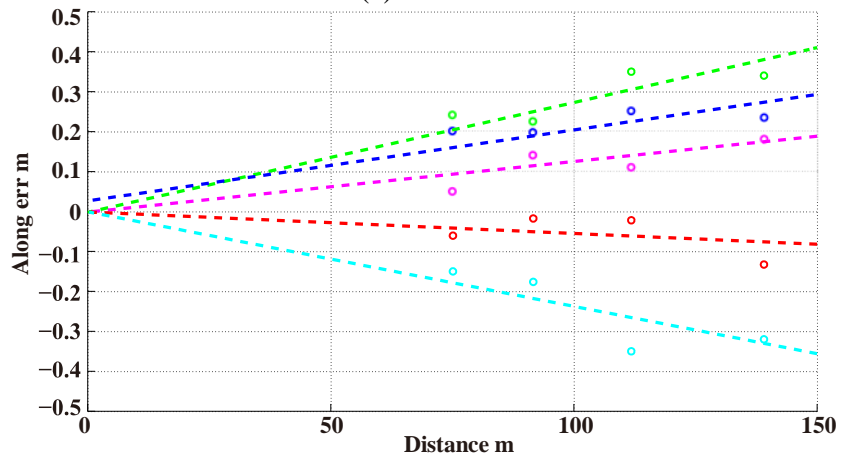

(b)Along error

Fig. 10 Position error of road images. 


\section{Road Marking Detection}

Road markings are detected from the rear view camera images. Extracted road markings are integrated to digital road map. Figre.1(C) shows an example that extracted Zebra zones are added on the lane level map. Two voting schemes are proposed and combined for accurate road marking position estimation and classification [15]. The first scheme is multi-frame voting with geometric features. In the voting, the camera image edge points and voted values are represented as tensors. The voting process is divided into ball voting and stick voting. Ball voting identifies the edge orientation, whereas stick voting reinforces the given orientation. The second scheme is road marking contour localization. Classification is based on the similarity between the fitted contour and the tensor field.
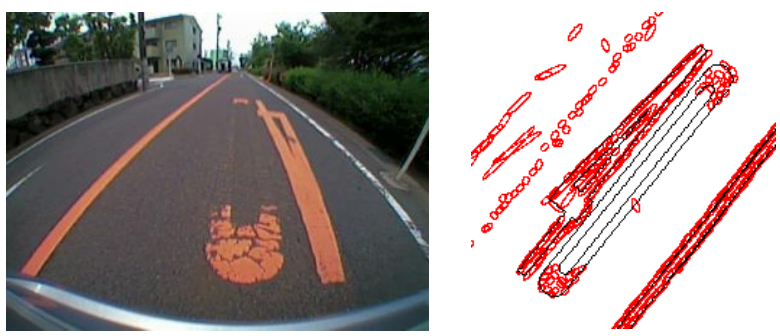

Fig. 11 Rear view camera image and tensor field.

Fig. 11 shows an example of a rear-view image with a faded speed limit mark, and its tensor field. This mark indicates that the speed limit is $40 \mathrm{~km} / \mathrm{h}$. In the voting process, tensors are projected to the real world coordinates. In Fig. 11, the red ellipses show the projected tensors and the black lines show the road mark contours. The $40 \mathrm{~km} / \mathrm{h}$ speed limit was detected from this tensor field. This road marking is identified by characteristic parts, even though the other parts were seriously degraded. This proposal enables a $91 \%$ correct detection and classification rate with four false detections per $100 \mathrm{~m}^{(16)}$.

In our proposal, orthographic road image are generated by several road image at each lane.Therefore, redundancy road marking results of each lane can be used to generate digital road map. Using redundancy detection result, road marking detection result of the digital road map, will be improved.

\section{Orthographic Road Image Generation}

Orthographic road images, which are similar to aerial images, are generated to connect road images. Fig. 12 and Fig. 13 show comparisons with commercial orthographic images and our proposal ones. In our proposal, orthographic road images are generated to use more than ten road images. When integrating these images, only the expansion and contraction based on image features are considered because the vehicle trajectory is accurate and the rotational error is small. Therefore, when combining road images, it is only necessary to consider expansion and contraction between road images. In addition, the proposed positioning technique $^{(3)}$ enables a limited matching search range. The positioning accuracy is 5 meters even in an urban area.

In Fig. 12 and Fig. 13, the proposed method automatically generates an orthographic image with a similar precision to that of

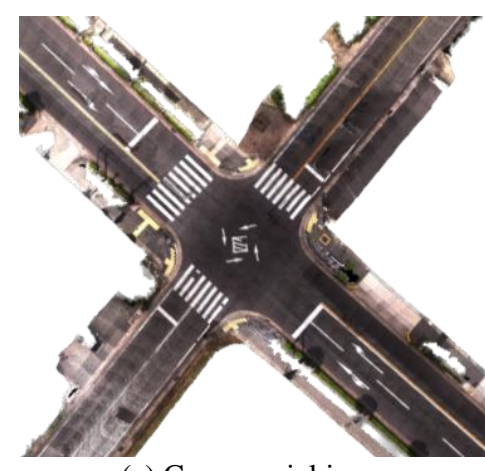

(a) Commercial image

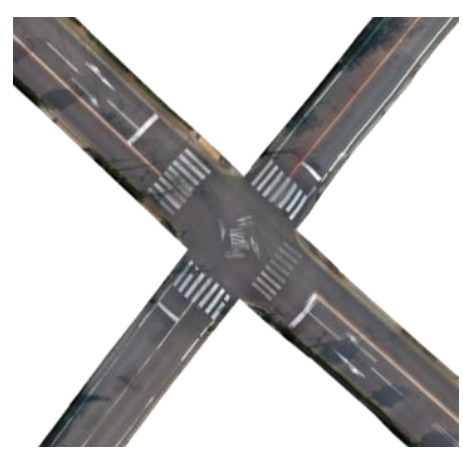

(b) Our proposal

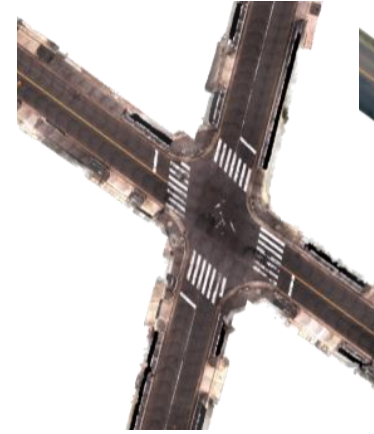

(c) Commercial image

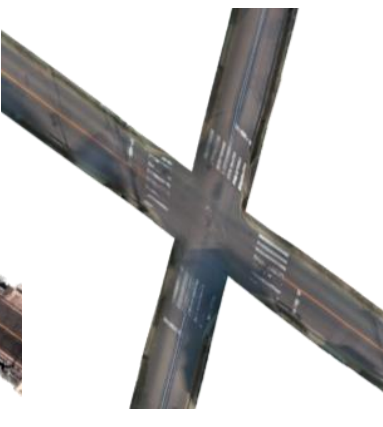

(d) Our proposal

Fig. 12 Comparison between commercial orthographic road image and our proposal of intersections. Resolution of each image is $5 \mathrm{~cm}$ per pixel.

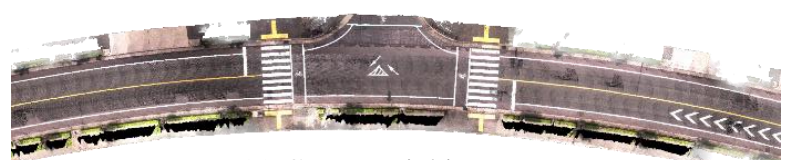

(a) Commercial image

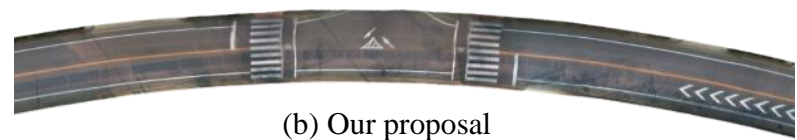

Fig. 13 Comparison between commercial ortho-image and our proposal of a curve road.

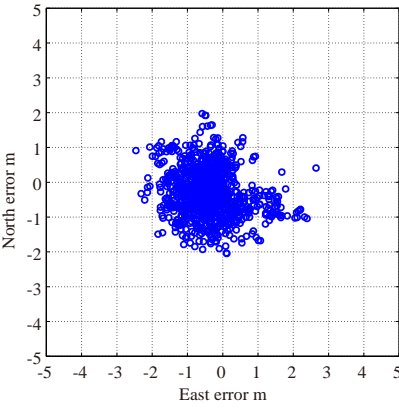

(a) Position error

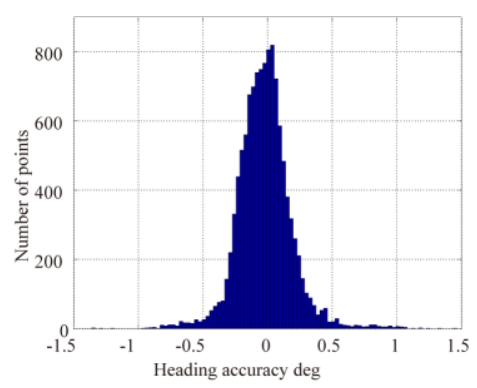

(b) Heading angle error
Fig. 14 Position and heading error. 


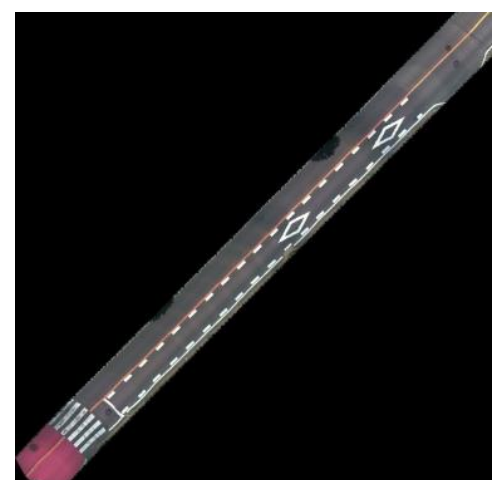

(a)Input Image

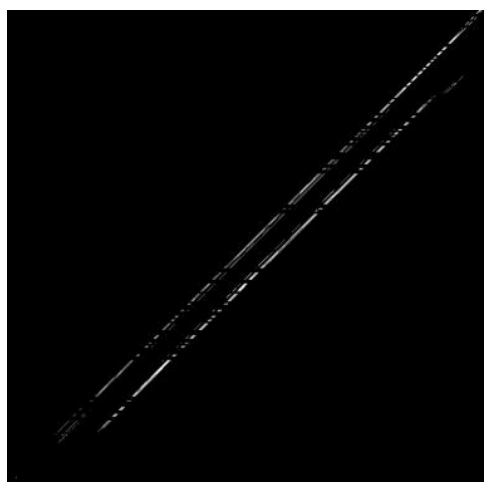

(b)Lane Boundary

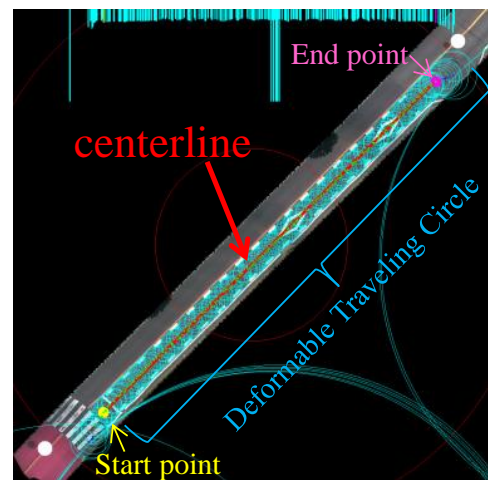

(c)Lane Centerline

Fig. 15 Example of lane graph construction of driving lane.

the survey level method of commercial ones. The resolution of Fig. 12 and Fig. 13 is $5 \mathrm{~cm}$. The accuracy of our orthographic road image depends on road images' accuracy that is shown in Fig. 10. Fig. 14 shows the positioning accuracy $(2.2 \mathrm{~m}$ : 2DRMS error) and heading accuracy $(0.21 \mathrm{deg}, \mathrm{SD})$ of the proposed method in this test. The true vehicle position, trajectory, and heading angles were measured by POSLV610 for evaluation ${ }^{(17)}$.

\section{Lane-Level Map Generation}

This section proposes an approach for generating lane-level maps, which show the fundamental properties of driving lanes, including the lane centerline, endpoints, lane width, lane curvature, and lane connectivity. This approach is based on the integrated orthograrfic road images and real vehicle trajectories. In the proposed system, a driving lane is represented by its centerline associated with additional information such as lane width, lane curvature, and the like. Such a representation is more suitable for vehicle navigation and ADAS applications. The lane centerlines are modeled as smooth curves, instead of dense center point clouds to reduce the immense amount of data required for storage and processing. The proposed system uses an approximated clothoid spline to model the normal roadways in a link segment, and employs cubic splines to generate the "virtual" transition driving lanes for intersections in a node segment ${ }^{(18)}$.

As shown in Fig. 15, given a local image in a link segment, the lane boundaries are detected first (Fig.15 (b)). Next, the centerline points are extracted using DTC (Deformable Traveling Circles based on vehicle trajectories. DTCs are estimated by expanding/shrinking circle size in the lane boundary. The mean and variance of the circle sizes are analyzed and the entry and exit points are determined by the turning points of the circle sizes. Once the centerline points are obtained, the RANdom Sample Consensus (RANSAC) algorithm is applied to fit the lane centerline based on the clothoid lane model in the link segment. After fitting the clothoid lane model, it is crucial to determine the entry and exit points for the driving lanes.

Fig. 16 shows an example lane-level map at an intersection. The colored lines show the centerline of each driving lane. The colored dots show the exit and entry points. Width and curvature are attached to the centerline. The example lane-map indicates that the proposed approach can extract the fundamental properties of the lane segments. In adittion, each exit and entry points is connected by sommothed path.

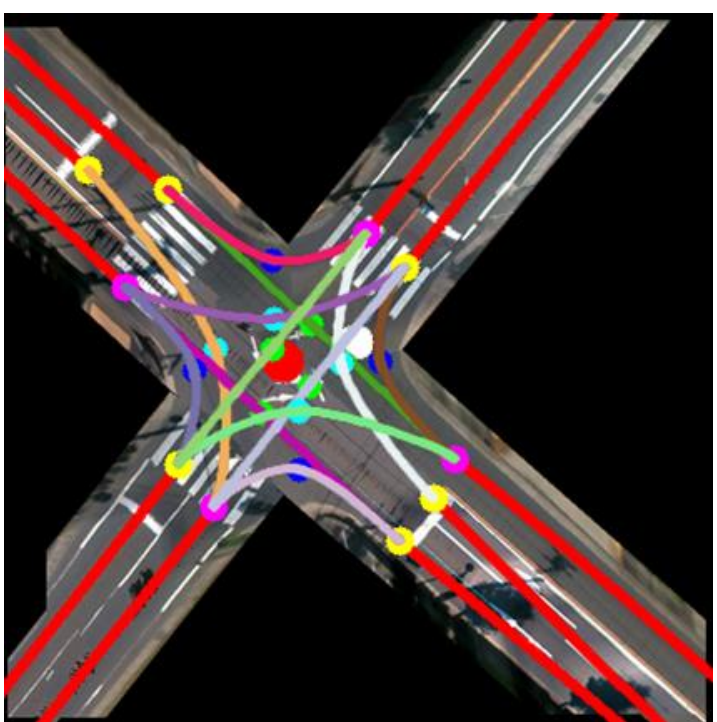

Fig. 16 Example of lane-level map at an intersection.

\section{Conclution}

This paper proposes a technique to generate orthographic road images and lane level maps using low-cost in-vehicle sensors. This proposal helps to reduce the cost of accurate digital maps for ADAS. The key feature of this method is the integration of vehicle driving data based on accurate trajectories and heading angles to generate digital maps. This paper describes the road image generation techniques and its systems. In addition, techniques are also being developed to detect road boundaries from accumulated depth information using a stereo camera. These results can be converted to digital maps suitable for ADAS. Digital maps are effective tools for driver support systems to enhance safety and comfort.

Furthermore, the reliability of the map is important to use the map in ADAS. If the map has error or wrong, it causes problems. In the proposed method, by using the probe technique, it will be possible to quickly construct and to check the reliability of the map. In adittion orthographic road image are generated by several road image at each lane. Wealth and redundancy data will improve digital road map accuracy and availability that based on road marking detection result. 


\section{References}

(1)M.Schlingelhof, D.Betaille, P.Bonnifait, K.Demaseure : Advanced Positioning Technologies for Co-operative Systems, Transactions on IEEE Intelligent Transportation Systems, Vol.2, No.2, pp.81-91. (2008)

(2)HS Tan, J Huang : DGPS-Based Vehicle-to-Vehicle Cooperative Collision Warning: Engineering Feasibility Viewpoints , Transactions on IEEE Intelligent Transportation Systems, Vol.7, No.4, pp.415 - 428 (2006)

(3)Julius Ziegler, Henning Lategahn, Markus Schreiber, Christoph G. Keller, Carsten Knöppel, Jochen Hipp, Martin Haueis and Christoph Stiller : Video Based Localization for BERTHA, IEEE Intelligent Vehicles Symposium (2014)

(4)Jun-ichi Meguro, Yoshiko Kojima, Noriyoshi Suzuki, Eiji Teramoto : Automotive Positioning Based on Bundle Adjustment of GPS Raw Data and Vehicle Trajectory, Proceedings of the 24th International Technical Meeting of The Satellite Division of the Institute of Navigation (ION GNSS 2011), pp. 1005- (2011)

(5)Kojiro Takeyama, Yoshiko Kojima, Eiji Teramoto : Trajectory Estimation Improvement Based on Time-series Constraint of GPS Doppler and INS in Urban Areas, Proceedings of IEEE/ION Position Location and Navigation Symposium (2012)

(6)Gontran H, Skaloud J, Gillieron PY : A mobile mapping system for road data capture via a single camera, Proceedings of sixth optical 3D measurement techniques (2003)

(7)Hunter G., Cox C., Kremer J. : Development of A Commercial Laser Scanning Mobile Mapping System StreetMapper , Processing of 2nd International Workshop of the future of Remote Sensing.(2006)

(8) Yanying Li, McDonald, M. : Link travel time estimation using single GPS equipped probe vehicle , Proceedings of the IEEE 5 th International Conference on Intelligent Transportation Systems (2002)

(9)Cyber-Navi introduction in Pioneer Corporation website (Japanese), http://pioneer.jp/carrozzeria/carnavi/cybernavi/ (access date 2015/3/2)

(10)Steven E. Shladover, Thomas M. Kuhn : Traffic Probe Data Processing for Full-Scale Deployment of VehicleInfrastructure Integration , Proceedings of the Intelligent Transportation Systems and Vehicle-Highway Automation (2008)

(11)Andreas Geiger : Monocular Road Mosaicing for Urban Environments, Proceedings of the Intelligent Vehicles Symposium (2009)

(12)Noda M., Takahashi T., Deguchi D., Ide I., Murase H., Kojima Y., Naito T., : Road Image Update using In-vehicle Camera Images and Aerial Image, Proceedings of the Intelligent Vehicles Symposium (2011)

(13)Yoshiko Kojima, Jun-ichi Meguro, Hiroyuki Ishida, Akihiro Watanabe, Noriyoshi Suzuki, Hiroshi Murase : Precise Localization using Tightly Coupled Integration based on Trajectory estimated from GPS Doppler, Proceedings of the International Symposium on Advanced Vehicle Control (2010)

(14)Jun-ichi Meguro, Hiroyuki Ishida, Kiyosumi Kidono, Yoshiko kojima : Road Ortho-Image Generation based on Accurate Vehicle Trajectory Estimation by GPS Doppler, Proceedings of the Intelligent Vehicle Symposium (2012)

(15)Jun-ichi Meguro, Jun-ichi Takiguchi, Takumi Hashizume, Ryusuke Kurosaki : Development of an Autonomous Mobile Surveillance System Using a Network-based RTK-GPS, Proceedings of the IEEE International Conference on Robotics and Automation (2005)

(16)Hiroyuki Ishida, Kiyosumi Kidono, Yoshiko kojima, Takashi Naito : Road marking recognition for map generation using sparse tensor voting, Proceedings of the 21st International Conference on Pattern Recognition (2012)

(17) "Applanix web site", http://www.applanix.com/ (access date 2015/3/2)

(18)Chunzhao Guo, Jun-ichi Meguro, Yoshiko Kojima, Takashi Naito : Automatic Lane-level Map Generation for Advanced Driver Assistance Systems using Low-cost Sensors, Proceedings of the 2014 IEEE International Conference on Robotics and Automation (2014) 